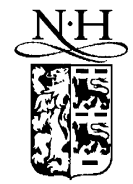

ELSEVIER

\title{
Exchange bias effects in ferromagnetic wires
}

\author{
M.K. Husain, A.O. Adeyeye*, C.C. Wang, V. Ng, T.S. Low \\ Information Storage Materials Laboratory, Department of Electrical and Computer Engineering, National University of Singapore, \\ 4 Engineering Drive 3, Singapore 117576, Singapore
}

Received 11 February 2003; received in revised form 18 March 2003

\begin{abstract}
We have investigated the exchange bias effect in micron-sized ferromagnetic wires made from Co and $\mathrm{Ni}_{80} \mathrm{Fe}_{20}$ films. The wires were fabricated using optical lithography, metallization by sputtering and lift-off technique. Magnetotransport measurements were performed at temperatures ranging from 3 to $300 \mathrm{~K}$. We observed marked changes in the magnetoresistance (MR) properties as the temperature is varied. At $300 \mathrm{~K}$, the field at which the sharp peak occurs corresponding to the magnetization reversal of the Co wires is $167 \mathrm{Oe}$ and is symmetrical about the origin. As the temperature was decreased to $3 \mathrm{~K}$, we observed a shift in the peak positions of the MR characteristics for both the forward and reverse field sweeps corresponding to a loop shift of $582 \mathrm{Oe}$ in the field axis. The asymmetric shift in the MR loops at low temperatures clearly indicates the exchange bias between ferromagnetic (Co) and antiferromagnetic parts (Co-oxide at the surfaces) from natural oxidation. $\mathrm{Ni}_{80} \mathrm{Fe}_{20}$ wires of the same geometry showed similar effect with a low exchange bias field. The onset of exchange biasing effect is found to be 70 and $15 \mathrm{~K}$ for the $\mathrm{Co}$ and $\mathrm{Ni}_{80} \mathrm{Fe}_{20}$ wires, respectively. A striking effect is the existence of exchange biasing effect from the sidewalls of the wires even when the wires were capped with Au film.
\end{abstract}

(C) 2003 Elsevier Science B.V. All rights reserved.

Keywords: Exchange bias; Magnetic wires; Magnetoresistance

\section{Introduction}

Laterally defined micron and sub-micron magnetic structures have received great attention because of the advances in lithographic and magnetic measurement techniques [1,2] as well as due to their potential for practical applications, for example, data storage devices [3]. Dramatic changes in magnetic properties such as coercivity, saturation that occur due to the change in lateral

\footnotetext{
*Corresponding author. Tel.: +65-68745071; fax: +6567791103.

E-mail address: eleaao@nus.edu.sg (A.O. Adeyeye).
}

dimensions provide the main motivation for studying these devices. A detail review of the general effects of patterning in ferromagnetic films can be found in Ref. [3] and references therein. One of the main properties that is of interest in magnetic ordered structures is the modification of magnetization reversal processes at low temperatures. One such phenomenon, "exchange bias" between ferromagnetic and antiferromagnetic materials, was first reported in ultrafine particles of Co with a cobaltous oxide shell [4]. This biasing effect was also observed in many different systems containing ferromagnetic (FM)-antiferromagnetic (AFM) interfaces [5-10]. The exchange bias 
properties of oxidized transition metal nanoparticles and films have been extensively studied [4, 11-13]. In an MR head the output response is not linear. Therefore an exchange bias is required for linearization, which can be achieved by coupling FM to an AFM [14]. Research on exchange biasing effect in ferromagnetic wires is therefore of interest because of its potential application in the information storage. Earlier works on magnetic wires were mostly concentrated on size dependence and orientation dependence on field $[2,15,16]$. Fraune et al. [17] have investigated the size dependence of exchange bias in $\mathrm{NiO} / \mathrm{Ni}$ nanostructures. They observed that for $\mathrm{NiO} / \mathrm{Ni}$ wires narrower than $3 \mu \mathrm{m}$, the exchange bias field significantly depends on the wire width. In another experiment, Otani et al. [18] have investigated the magnetization reversal processes of the $\mathrm{Fe}_{19} \mathrm{Ni}_{81} /$ $\mathrm{NiO}$ sub-micron exchange coupled bilayer wires. They observed a size effect on the exchange coupling field when the wire is finer than $1 \mu \mathrm{m}$. Recently, exchange bias effect in patterned FeMn/ $\mathrm{NiFe}$ bilayer wire array have been investigated by Guo et al. [19].

In the present work, we have studied the magnetization reversal of array of lithographically defined micron sized $\mathrm{Co}$ and $\mathrm{Ni}_{80} \mathrm{Fe}_{20}$ wires at temperatures ranging from 3 to $300 \mathrm{~K}$. As we reduced the temperature beyond a critical point, we observed a shift in the peak positions of the MR characteristics for both the forward and reverse field sweeps. This asymmetric shift in the MR loops at low temperatures clearly indicates the exchange bias between the ferromagnetic and antiferromagnetic components. The latter appears due to the formation of antiferromagnetic Cooxides and $\mathrm{Ni}_{80} \mathrm{Fe}_{20}$ oxides at the surfaces of the corresponding FM wires during fabrication.

\section{Experimental procedures}

The magnetic wires were fabricated on $\mathrm{Si}\left(\begin{array}{lll}0 & 0 & 1\end{array}\right)$ substrates using the optical lithography, metallization and lift-off techniques. A base pressure of $3 \times 10^{-7}$ Torr was maintained during sputtering of the magnetic materials at room temperature. The working pressure and Ar gas flow rate during film

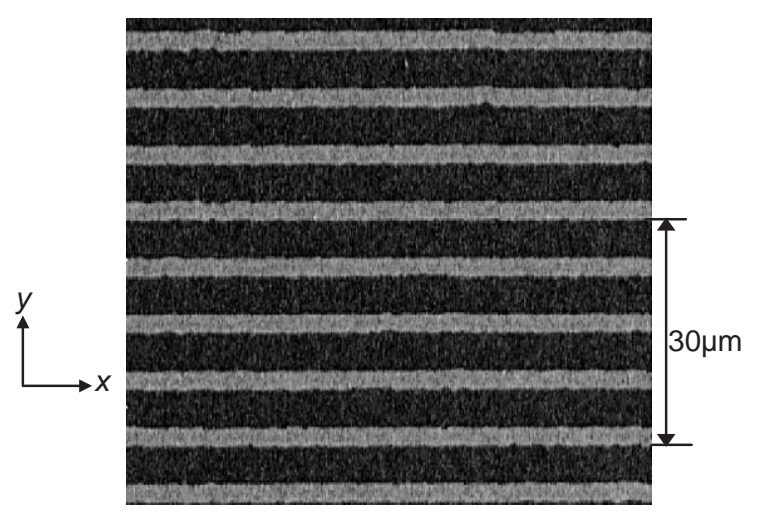

Fig. 1. Scanning electron micrograph of Co wires with width, $w=2.5 \mu \mathrm{m}$ and spacing, $s=5 \mu \mathrm{m}$.

deposition were set at $10 \mathrm{mTorr}$ and 10 standard $\mathrm{cm}^{3} / \min (\mathrm{sccm})$, respectively, and a DC power of $100 \mathrm{~W}$ was used. The wires are each of width $w=2.5 \mu \mathrm{m}$, spacing $s=5 \mu \mathrm{m}$, thickness $t=$ $40 \mathrm{~nm}$, length $l=4 \mathrm{~mm}$ and extend over a distance of $4 \mathrm{~mm}$. Electrical contacts were made using a combination of standard optical lithography, metallization and lift-off of $150 \mathrm{~nm} \mathrm{Al}$. The contacts were made parallel to the wire axis. A scanning electron micrograph of the array of $\mathrm{Co}$ $(40 \mathrm{~nm})$ wires is shown in Fig. 1. For MR measurements a DC sense current of $1 \mathrm{~mA}$ was passed through the current leads and the resistance was recorded automatically using a four terminal method as the in plane magnetic field was swept. The field was applied perpendicular to the wire axis. The MR measurements were performed at temperatures ranging from 3 to $300 \mathrm{~K}$. The low temperature measurements were performed using a Janis Model SVT Research Cryostat that enabled a temperature variation from $1.5 \mathrm{~K}$ to room temperature.

\section{Results and discussion}

Fig. 2 shows the MR curves for $40 \mathrm{~nm}$ thick Co wires when the applied field is perpendicular to the wire long axis for various temperatures. First, a negative field sufficiently large to saturate the wires was applied perpendicular to the wire axis. As the magnetic field was swept back toward a positive 


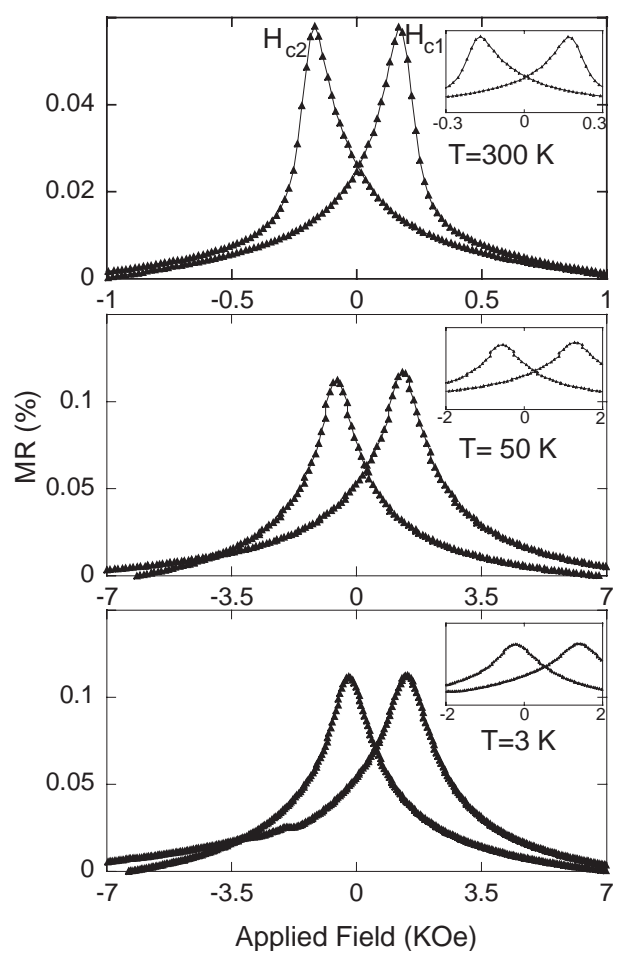

Fig. 2. MR curves of Co wire array for various temperatures with field applied perpendicular to the wire axis. The low field region is magnified in the inset.

value at constant rate, the MR increased until it reached the peak $\left(H_{\mathrm{cl}}\right)$ when the magnetization of the ferromagnetic wires switched its direction and tend to align with the field. In a similar fashion, the peak $\left(H_{\mathrm{c} 2}\right)$ on the MR curve was obtained after applying a large positive field, and then sweeping the field towards negative values. The crossover at large negative fields could be due to a slight drift in temperature during measurement. The peak positions $\left(H_{\mathrm{c} 1}\right.$ and $\left.H_{\mathrm{c} 2}\right)$ shown in Fig. 2 in the MR curves is due to AMR effect corresponding to the switching of magnetization in $\mathrm{Co}$ wires. We observed that the peak positions shift noticeably with temperature.

Table 1 shows the field values at which the peaks $\left(H_{\mathrm{c} 1}\right.$ and $H_{\mathrm{c} 2}$ ) occur in the MR characteristics for $\mathrm{Co}$ wires at different temperatures. At $300 \mathrm{~K}$ the peaks are symmetrical around the origin with a switching field $167 \mathrm{Oe}$. As the temperature is decreased we observed an unusual asymmetry in
Table 1

The peak positions on the MR curves of Co wires at various temperatures

\begin{tabular}{lllllll}
\hline $300 \mathrm{~K}$ & & $50 \mathrm{~K}$ & & $3 \mathrm{~K}$ & \\
\cline { 1 - 2 } \cline { 5 - 6 } & $H_{\mathrm{c} 1}$ & $H_{\mathrm{c} 2}$ & $H_{\mathrm{c} 1}$ & $H_{\mathrm{c} 2}$ & $H_{\mathrm{c} 2}$ \\
\hline $167 \mathrm{Oe}$ & $-167 \mathrm{Oe}$ & $1250 \mathrm{Oe}$ & $-638 \mathrm{Oe}$ & & $1400 \mathrm{Oe}$ & $-236 \mathrm{Oe}$ \\
\hline
\end{tabular}

the peak positions for the forward and backward field sweeps. The peaks in the MR loops shifts asymmetrically to higher positive fields. The asymmetric shift in the peak positions of the MR loops at low temperatures indicates the appearance of exchange bias $\left(H_{\mathrm{E}}\right)$ in the system. The biasing occurred due to the coupling of spins in the FM and AFM when cooled below Néel temperature $\left(T_{\mathrm{N}}\right) . \quad H_{\mathrm{E}}$ depends strongly on the spin structure at the interface [8-10].

Shifting of the fields to higher fields corresponds to the increase of coercivity with decreasing temperature. The overall coercivity is defined as

$H_{\mathrm{C}}=\frac{1}{2}\left|H_{\mathrm{c} 1}-H_{\mathrm{c} 2}\right|$,

where $H_{\mathrm{c} 1}$ and $H_{\mathrm{c} 2}$ are the coercive fields corresponding to the right and left of the MR loops.

The asymmetric shift in peak positions is best described in terms of an effective internal "exchange field" $H_{\mathrm{E}}$, which arises from the FM-AFM interface, is defined as [12]

$H_{\mathrm{E}}=\frac{1}{2}\left|H_{\mathrm{c} 1}+H_{\mathrm{c} 2}\right|$.

For Co wires, when the temperature is $50 \mathrm{~K}$, an exchange bias field of $306 \mathrm{Oe}$ is measured. For $T=3 \mathrm{~K}$, we measured a bias field of $582 \mathrm{Oe}$.

Similar measurements were performed on $\mathrm{Ni}_{80} \mathrm{Fe}_{20}$ wires having the same geometry and fabricated under similar conditions as Co wires. Representative MR curves at various temperatures are shown in Fig. 3. We also observed an asymmetric shift in peaks in the MR characteristics at low temperatures. However, the magnitude of the shift is small when compared with that observed in Co wires of the same lateral dimensions. During the fabrication process, the ferromagnetic wires were exposed to the atmosphere 


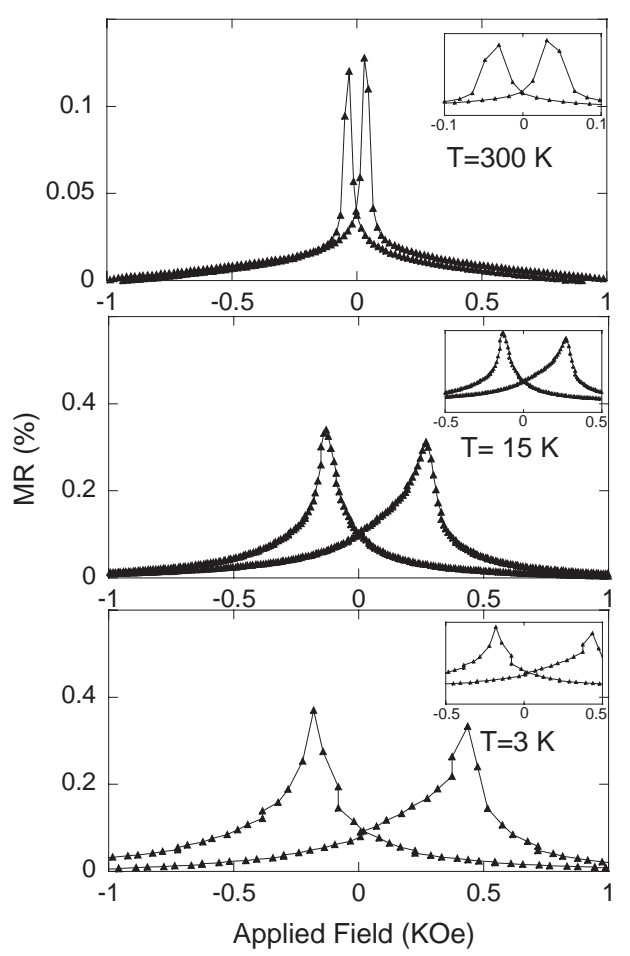

Fig. 3. MR curves of $\mathrm{Ni}_{80} \mathrm{Fe}_{20}$ wire array for various temperatures with field applied perpendicular to the wire axis. The low field region is magnified in the inset.

before measurements resulting in the natural oxidation of the wires top surface and sidewalls.

We also observed in Fig. 3 (for $T<15 \mathrm{~K}$ ) that the forward and reverse field loops are asymmetric in shape and the peak height is different. This asymmetry is not an artifact of sample misalignment, or the current direction misalignment as the asymmetry disappears when the sample is no longer exchange biased at $300 \mathrm{~K}$. This effect has been observed by Leighton et al. [20] and can be attributed to the asymmetrical magnetization reversal.

In order to determine the onset of exchange bias effect in both $\mathrm{Co}$ and $\mathrm{Ni}_{80} \mathrm{Fe}_{20}$ wires we have performed a systematic study on the asymmetric shift in the peaks as a function of temperature. Shown in Fig. 4 is the temperature dependence of the exchange bias field for both the $\mathrm{Co}$ and $\mathrm{Ni}_{80} \mathrm{Fe}_{20}$ ferromagnetic wires. For $T<100 \mathrm{~K}$ we observed that the peak positions are symmetric about the origin. As the temperature was reduced

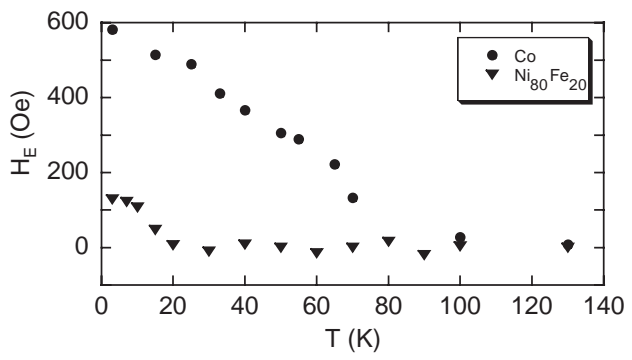

Fig. 4. Exchange bias field $H_{\mathrm{E}}$ vs. temperature for Co and $\mathrm{Ni}_{80} \mathrm{Fe}_{20}$ wires.

further to $70 \mathrm{~K}$ a marked shift in the peak position was observed for Co wires. From the asymmetric shift of the peak positions we were able to determine the exchange bias field of 133 Oe. Further decrease in temperature resulted in a marked exchange bias field. The blocking temperature $\left(T_{\mathrm{B}}\right)$ of the antiferromagnetic Co-oxide at the wire edges estimated from Fig. 4 is $\sim 70 \mathrm{~K}$. We have measured the atomic composition of the oxide on the top surface using X-ray photoelectron spectroscopy (XPS). We observed the existence of $\mathrm{CoO}$ and $\mathrm{Co}_{3} \mathrm{O}_{4}$ on the surface. Therefore, possible antiferromagnetic components in our sample could be $\mathrm{CoO}$ and $\mathrm{Co}_{3} \mathrm{O}_{4}$. Bulk $\mathrm{Co}_{3} \mathrm{O}_{4}$ and $\mathrm{CoO}$ have $T_{\mathrm{N}}$ of about $30 \mathrm{~K}[21]$ and $290 \mathrm{~K}$ $[13,22]$, respectively. It has been observed by Lund et al. [23] that $T_{\mathrm{B}}$ depends strongly on the thickness and anisotropy of the AFM. Therefore the large difference between $T_{\mathrm{B}}$ and $T_{\mathrm{N}}$ of Cooxides in our sample may be due to low thickness or low anisotropy (because of defects) of the AFM. There might also be some contribution from other AFM phases [7,10].

For $\mathrm{Ni}_{80} \mathrm{Fe}_{20}$ wires, however, exchange-biasing fields of 48 and 128 Oe were measured when the temperature is reduced from 15 to $3 \mathrm{~K}$, respectively. This is quite in agreement with similar work done by O'Grady et al. [24] on NiFe layers and $\mathrm{NiFe} / \mathrm{Cr}$ multilayers. The possible candidates for AFM in $\mathrm{Ni}_{80} \mathrm{Fe}_{20}$ are the oxides of $\mathrm{Fe}$ or Ni. This, however, could not be readily established from our XPS measurements. The Néel temperatures $\left(T_{\mathrm{N}}\right)$ of $\mathrm{NiO}$ and $\mathrm{FeO}$ are 523 and $198 \mathrm{~K}$, respectively and therefore the most likely component is $\mathrm{FeO}$. Nevertheless, it is clear that only ferromagnetic parts contribute to magnetization at $20 \mathrm{~K}$. This is 
consistent as the exchange bias disappears above $20 \mathrm{~K}$.

Shown in Fig. 5 is a plot of the coercivity $\left(H_{\mathrm{C}}\right)$ measured from the peak positions as a function of temperature ( $T$ ) for both $\mathrm{Co}$ and $\mathrm{Ni}_{80} \mathrm{Fe}_{20}$ wires. We observed a marked increase in $H_{\mathrm{C}}$ as $T$ is decreased. This increase in $H_{\mathrm{C}}$ below $T_{\mathrm{N}}$ can be intuitively explained. When the FM spin switches its direction it irreversibly drags the AFM spins thus increasing the FM coercivity. However, direct dependence on temperature is difficult to understand because $H_{\mathrm{C}}$ is also affected by the microstructures of FM layer that changes from system to system $[10,25,26]$.

$\mathrm{Ni}_{80} \mathrm{Fe}_{20}$ wires show an unusual effect when we compare $H_{\mathrm{C}}\left(T_{\mathrm{B}} \sim 50 \mathrm{~K}\right)$ and $H_{\mathrm{E}}$ curves of Figs. 5 and 4 , respectively. The difference in $T_{\mathrm{B}}$ may be due to coercivity enhancement above $T_{\mathrm{B}}$ as observed by Leighton et al. [27] which is attributed to the spin fluctuations in the AFM layer inducing additional anisotropy in the FM layer.

In order to investigate the contribution of the wire sidewalls to the exchange bias field, we fabricated an array of Co wires of the same lateral dimensions and under the same fabrication conditions, except that we capped the wires top surface with $5 \mathrm{~nm}$ of $\mathrm{Au}$ to prevent oxidation. The sidewalls of the wires were allowed to oxidize naturally. Fig. 6 shows corresponding MR response at $3 \mathrm{~K}$. We observed that the asymmetric shift exists in the peaks in the MR loop though it is very small compared to the one in Fig. 2. The exchange bias field at $3 \mathrm{~K}$ is $22 \mathrm{Oe}$ compared with an exchange bias field of 582 Oe for the sample without $\mathrm{Au}$ capping. While the exchange biasing

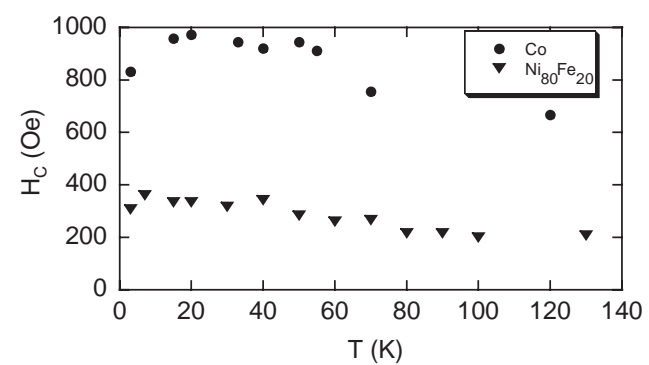

Fig. 5. Coercivity $H_{\mathrm{C}}$ vs. temperature for $\mathrm{Co}$ and $\mathrm{Ni}_{80} \mathrm{Fe}_{20}$ wires.

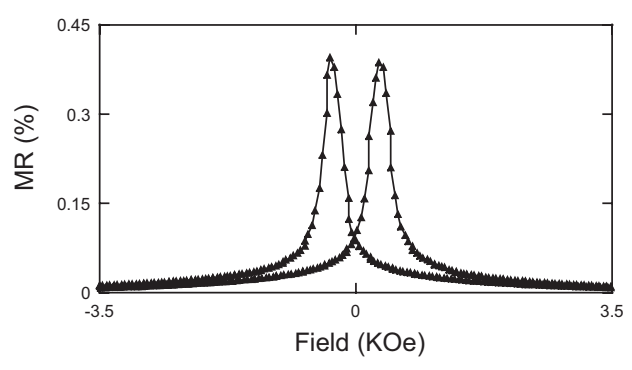

Fig. 6. MR curve at $T=3 \mathrm{~K}$ for Co $(40 \mathrm{~nm}) / \mathrm{Au}(5 \mathrm{~nm})$ wires with field applied perpendicular to wire axis.
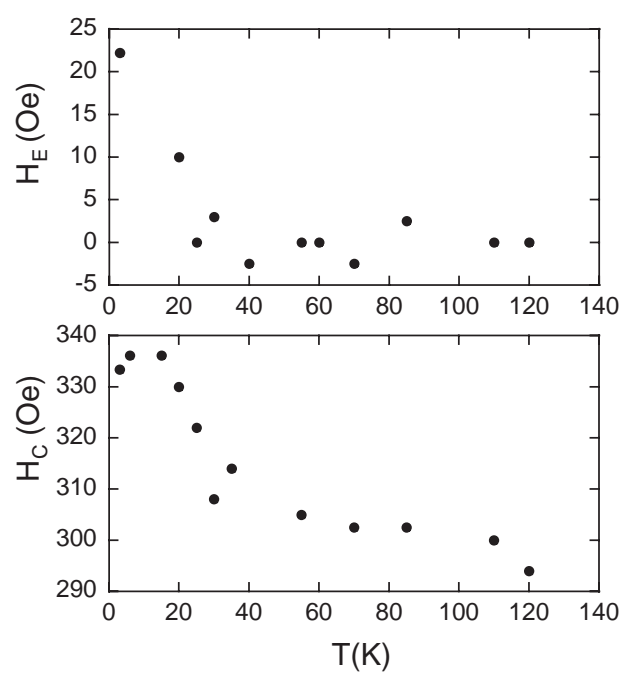

Fig. 7. $H_{\mathrm{E}}, H_{\mathrm{C}}$ vs. temperature for Co wire array capped with $\mathrm{Au}(5 \mathrm{~nm})$.

effect from the top surface of the wires observed in Fig. 2 may not be new, the effect found in capped wires shown in Fig. 6 is very striking and surprising.

$H_{\mathrm{C}}$ and $H_{\mathrm{E}}$ is plotted in Fig. 7 as a function of temperature for the Au capped $\mathrm{Co}$ wire array. $T_{\mathrm{B}}$ is found to be $30 \mathrm{~K}$ which is much lower than what was observed in the uncapped wires. $H_{\mathrm{C}}$ values at different temperatures are also observed to be much lower in capped wires. This can be attributed to the comparatively less effective AFM spins in the capped wires due to the absence of contribution of exchange bias from the top surface of the wires. 


\section{Conclusion}

We have investigated the temperature-dependent MR properties of arrays of micron-sized Co and $\mathrm{Ni}_{80} \mathrm{Fe}_{20}$ wires. We observed an exchange biasing effect at low temperatures manifested by an asymmetric shift in the peak positions on their MR characteristics. We have attributed this to the presence of antiferromagnetic material at the surfaces of the wires due to the natural oxidation of the magnetic materials during fabrication process. The onset of exchange biasing effect is found to be 70 and $15 \mathrm{~K}$ for the Co wires and $\mathrm{Ni}_{80} \mathrm{Fe}_{20}$ wires, respectively. The exchange biasing effect was observed even when the wires top surfaces were capped with Au.

\section{Acknowledgements}

This work was supported by the National University of Singapore (NUS) under grant R263-000-180-112. M.K.H. would like to thank NUS for their Research Scholarship.

\section{References}

[1] A. Maeda, M. Kume, T. Ogura, K. Kuroki, T. Yamada, N. Nishikawa, Y. Harada, J. Appl. Phys. 76 (1994) 6667.

[2] A.O. Adeyeye, J.A.C. Bland, C. Daboo, D.G. Hasko, Phys. Rev. B 56 (1997) 3265;

A.O. Adeyeye, G. Lauhoff, J.A.C. Bland, C. Daboo, D.G. Hasko, H. Ahmed, Appl. Phys. Lett. 70 (1997) 1046.

[3] J.I. Martín, J. Nogués, K. Liu, J.L. Vicent, I.K. Schuller, J. Magn. Magn. Mater. 256 (2003) 449.

[4] W.H. Melklejohn, C.P. Bean, Phys. Rev. 102 (1957) 1413.

[5] T.J. Moran, J.M. Gallego, I.K. Schuller, J. Appl. Phys. 78 (1995) 1887.

[6] R. Jungblut, R. Coehoorn, M.T. Johnson, J. aan de Stegge, A. Reinders, J. Appl. Phys. 75 (1994) 6659.
[7] M. Takahashi, A. Yanai, S. Taguchi, T. Suzuki, Jpn. J. Appl. Phys. 19 (1980) 1093.

[8] M. Kiwi, J. Magn. Magn. Mater. 234 (2001) 584.

[9] A.E. Berkowitz, K. Takano, J. Magn. Magn. Mater. 200 (1999) 552.

[10] J. Nogués, I.K. Schuller, J. Magn. Magn. Mater. 192 (1999) 203.

[11] S. Gangopadhyay, G.C. Hajipanayis, B. Dale, C.M. Sorensen, K.J. Klabunde, V. Papaeftymiou, A. Kostikas, Phys. Rev. B 45 (1992) 9778.

[12] S. Spagna, M.B. Maple, R.E. Sager, J. Appl. Phys. 79 (1996) 4926.

[13] L. Smardz, U. Köbler, W. Zinn, J. Appl. Phys. 71 (1992) 5199.

[14] J.C.S. Kools, IEEE Trans. Magn. 32 (1996) 3165.

[15] A.O. Adeyeye, J.A.C. Bland, C. Daboo, J. Lee, U. Ebels, H. Ahmed, J. Appl. Phys. 79 (1996) 6120;

A.O. Adeyeye, R.P. Cowburn, M.E. Welland, J. Magn. Magn. Mater. 213 (2000) L1.

[16] C. Shearwood, S.J. Blundell, M.J. Baird, J.A.C. Bland, M. Gester, H. Ahmed, H.P. Hughes, J. Appl. Phys. 75 (1994) 5249.

[17] M. Fraune, U. Rüdiger, G. Güntherodt, S. Cardoso, P. Freitas, Appl. Phys. Lett. 77 (2000) 3815.

[18] Y. Otani, A. Nemoto, S.G. Kim, K. Fukamichi, O. Kitakami, Y. Shimada, J. Magn. Magn. Mater. 198-199 (1999) 434.

[19] Z.B. Guo, K.B. Li, G.C. Han, Z.Y. Liu, P. Luo, Y.H. Wu, J. Magn. Magn. Mater. 251 (2002) 323.

[20] C. Leighton, M. Song, J. Nogués, M.C. Cyrille, I.K. Schuller, J. Appl. Phys. 88 (2000) 344.

[21] W. Kundig, M. Kobelt, A. Appel, G. Constabaris, R.H. Lindequist, J. Phys. Chem. Solids 30 (1969) 819.

[22] M.D. Rechtin, B.L. Averbach, Phys. Rev. B 5 (1972) 2693.

[23] M.S. Lund, W.A.A. Macedo, K. Liu, J. Norgués, I.K. Schuller, C. Leighton, Phys. Rev. B 66 (2002) 054422.

[24] K. O'Grady, S.J. Greaves, S.M. Thompson, J. Magn. Magn. Mater. 156 (1996) 253.

[25] M. Tsunoda, Y. Tsuchuya, M. Konoto, M. Takahashi, J. Magn. Magn. Mater. 171 (1997) 29.

[26] R.P. Michel, A. Chaiken, Y.K. Kim, L.E. Johnson, IEEE Trans. Magn. 32 (1996) 4651.

[27] C. Leighton, H. Suhl, M.J. Pechan, R. Compton, J. Nogués, I.K. Schuller, J. Appl. Phys. 92 (2002) 1483. 